\title{
QUEEN'S
UNIVERSITY
BELFAST
}

\section{Temperature-dependent gap edge in strong-coupling superconductors determined using the Eliashberg-Nambu formalism}

Zheng, X-H., \& Walmsley, D. G. (2008). Temperature-dependent gap edge in strong-coupling superconductors determined using the Eliashberg-Nambu formalism. Physical Review B (Condensed Matter), 77(10), 104510-1104510-11. [104510]. https://doi.org/10.1103/PhyRevB.77.104510,

https://doi.org/doi.org/10.1103/PhysRevB.77.104510

Published in:

Physical Review B (Condensed Matter)

Queen's University Belfast - Research Portal:

Link to publication record in Queen's University Belfast Research Portal

\section{General rights}

Copyright for the publications made accessible via the Queen's University Belfast Research Portal is retained by the author(s) and / or other copyright owners and it is a condition of accessing these publications that users recognise and abide by the legal requirements associated with these rights.

Take down policy

The Research Portal is Queen's institutional repository that provides access to Queen's research output. Every effort has been made to ensure that content in the Research Portal does not infringe any person's rights, or applicable UK laws. If you discover content in the Research Portal that you believe breaches copyright or violates any law, please contact openaccess@qub.ac.uk. 


\title{
Temperature-dependent gap edge in strong-coupling superconductors determined using the Eliashberg-Nambu formalism
}

\begin{abstract}
X. H. Zheng* and D. G. Walmsley
Department of Physics and Astronomy, Queen's University of Belfast, Belfast BT7 1NN, Northern Ireland

(Received 14 November 2007; revised manuscript received 6 February 2008; published 10 March 2008)

Using the theory of Eliashberg and Nambu for strong-coupling superconductors, we have calculated the gap function for a model superconductor and a selection of real superconductors including the elements $\mathrm{Al}, \mathrm{Sn}, \mathrm{Tl}$, $\mathrm{Nb}, \mathrm{In}, \mathrm{Pb}$, and $\mathrm{Hg}$ and one alloy, $\mathrm{Bi}_{2} \mathrm{Tl}$. We have determined the temperature-dependent gap edge in each and found that in materials with weak electron-phonon coupling $(\lambda<0.8)$, it is single valued, but in materials with intermediate coupling $(0.8 \leqslant \lambda \leqslant 1.2)$, the gap edge is double valued. In materials with strong coupling ( $\lambda$ $>1.20$ ), not only is the gap edge double valued but it also departs significantly from the BCS form and develops a shoulderlike structure which may, in some cases, denote a gap edge exceeding the $T=0$ value. These computational results support the insights obtained by Leavens in an analytic consideration of the general problem. Both the shoulder and double value arise from a common origin seated in the form of the gap function in strongly coupled materials at finite temperatures. From the calculated gap function, we can determine the densities of states in the materials and the form of the tunneling current-voltage characteristics for junctions with these materials as electrodes. By way of illustration, results are shown for the contrasting cases of Sn $(\lambda=0.74)$ and $\mathrm{Hg}(\lambda=1.63)$. The reported results are distinct in several ways from BCS predictions and provide an incentive for further discriminative experimental studies with techniques such as tunneling and far infrared absorption.
\end{abstract}

DOI: 10.1103/PhysRevB.77.104510

PACS number(s): 74.20.Fg, 74.20.-z, 02.60.Cb

\section{INTRODUCTION}

The Eliashberg or Eliashberg-Nambu formalism for the theoretical description of superconductivity ${ }^{1,2}$ stems from the work by $\mathrm{Gokov}^{3}$ and $\mathrm{Migdal}^{4}$ and was developed into its present form by Schrieffer et al. ${ }^{5}$ Wada, ${ }^{6}$ and Ambegaokar and Tewordt. $^{7}$ This formalism has widely supplanted the original theory of superconductivity by Bardeen et al. ${ }^{8}$ (BCS) and has been extensively used to analyze novel strong-coupling superconductors. For example, it was used to study high temperature superconductivity with a pairing mechanism based jointly on electron-phonon and electronplasmon interactions. ${ }^{9}$ It was also used to study fullerenes, ${ }^{10}$ $\mathrm{C}_{60}$ in particular, ${ }^{11}$ quasi-two-dimensional metals, ${ }^{12}$ magnesium diboride, ${ }^{13-16} \mathrm{~s}$-wave narrow band superconductors, ${ }^{17}$ $\mathrm{Na}_{x} \mathrm{CoO}_{2} \cdot y \mathrm{H}_{2} \mathrm{O},{ }^{18}$ boron under pressure, ${ }^{19} \mathrm{MgCNi}_{3},{ }^{20}$ halogen doped carbon clathrates, ${ }^{21}$ and so forth. Very recently, it was extended to extract the spectrum of the effective electron-phonon interaction, $\alpha^{2}(\nu) F(\nu)$, from optical conductivity data. ${ }^{22}$

The early success of the Eliashberg-Nambu formalism rested on its acclaimed elucidation of the frequencydependent gap function at temperatures near zero. Extending the theory to higher temperatures opens up the opportunity to scrutinize it for possible limitations or disagreement with experimental results. To this end, we wish to call attention to the interesting discovery by Leavens that in the EliashbergNambu formalism, the edge of the superconductive energy gap can have two distinct values at the same temperature. ${ }^{23}$ The energy gap edge is defined as the value of the electron frequency $\omega$ (or electron energy, $\hbar=1$ customarily in the literature), satisfying the following relation:

$$
\mathfrak{R}[\Delta(\omega, T)]=\omega,
$$

where $\Delta(\omega, T)$ is the energy gap function (solution to the Eliashberg equations) or superconductive order parameter. In the analysis of Leavens, this double-valued gap edge arises from the particular $\omega$ dependence of $\Delta$ occurring in certain situations, which renders Eq. (1) into the form $\omega \propto \omega^{2}$ for small $\omega$, leading to a small but nonzero value of $\omega$ satisfying Eq. (1), in addition to its usual solution (at higher $\omega$ ) for the gap edge. This double value represents a significant departure from BCS behavior and was attributed to the breakdown of the quasiparticle approximation. ${ }^{23}$ Later, in 1991, Marsiglio and Carbotte commented that the double-valued gap edge is an artifact of the definition in Eq. (1): the true order parameter does indeed approach zero and is single valued as $T \rightarrow T_{c}$, where $T_{c}$ is the transition temperature. This vanishing of the order parameter occurs in a temperature range in which Eq. (1) does not have a solution. ${ }^{24}$

Marsiglio and Carbotte also discovered that, in addition to becoming double valued at relatively high temperatures, the upper value of the gap edge in the Eliashberg-Nambu formalism starts to depart considerably from the BCS value at $T \sim 0.1 T_{c}$ for very strong electron-phonon coupling $(\lambda$ $=100) .{ }^{24}$ Here, $\lambda$ is defined as

$$
\lambda=2 \int_{0}^{\omega_{M}} \alpha^{2}(\nu) F(\nu) \frac{d \nu}{\nu}
$$

and is the dimensionless electron-phonon coupling constant, $\nu$ the phonon frequency, and $\omega_{M}$ its maximum value. In contrast to the double-valued gap edge discovered by Leavens, this was not considered to be an artifact. $^{24}$ The question arises as to whether there is a common origin behind these two discoveries. If so, is this origin indeed related to the 
breakdown of the quasiparticle approximation? Faced with the different outcomes from the Eliashberg formalism and BCS theory, should we trust one rather than the other? Do we have to make this choice in real materials with realistic values of $\lambda$ ? These questions are worth considering and, to our knowledge, have neither been raised nor answered in the literature.

In this paper, we show that the double values of the gap edge in the Eliashberg-Nambu formalism and departure of the gap edge from the BCS curve of temperature dependence are of the same origin: they both arise from a sudden and significant drop of the gap function, $\Delta(\omega, T)$, for small $\omega$ at finite temperatures. At a more fundamental level, these effects are deduced to arise from two specific terms in the kernel of the Eliashberg equations, which are suppressed at $T=0$ but become increasingly important with increasing $T$, with apparently more significant impact in the case of strong electron-phonon interaction. We show that while the lower value of the gap edge can be suppressed by a technical treatment to imitate the experimental procedure for determining the gap, the curve of gap temperature dependence, or temperature curve for short, still displays a shoulderlike structure, marking a genuine departure from the BCS curve. "Shoulder" in this context means a peak above the BCS curve, with a maximum value sometimes exceeding even the value of the gap edge at $T=0$.

We observe through numerous examples, including a model superconductor, a range of real superconductive elements, and an alloy, the departure of the gap edge in the Eliashberg-Nambu formalism from the BCS prediction. We find that this departure takes place only at relatively high temperatures and in strong-coupling superconductors, with a realistic threshold of $\lambda>0.8$. As an important specific example, we study mercury in detail. The electron-phonon interaction in mercury is strong enough $(\lambda=1.63)$ to allow us to observe significant departure between the Eliashberg formalism and BCS theory. The experimentally measured temperature curve was shown to obey the BCS prediction almost perfectly. ${ }^{25}$ We argue that the Eliashberg-Nambu formalism is subject to the limitations of the quasiparticle approximation and ask if we may be seeing here the impact of the breakdown of the quasiparticle regime.

The paper is arranged as follows. In Sec. II, we describe a model superconductor, where the effective electron-phonon interaction is represented by a single peak, continuous with respect to phonon frequency and with continuous first order derivative. In Sec. III, the energy gap function of the model superconductor is calculated from the Eliashberg formalism and shown to have a double-valued edge when $\lambda>1$, as well as a shoulderlike structure in the temperature curve; both are shown to have the same origin. In Sec. IV, we imitate the experimental procedure for measuring the energy gap. We show that while the double values of the gap edge vanish in this procedure, the shoulderlike structure is still there. In Sec. $\mathrm{V}$, we study strong-coupling superconductive elements and an alloy and confirm that lead, mercury, and $\mathrm{Bi}_{2} \mathrm{Tl}(\lambda$ $>1.2$ ) may have both the double-valued gap edge and a shoulder in the temperature curve. In Sec. VI, we study moderately strongly coupled superconductive elements. We show that the gap edge may become double valued for both nio-

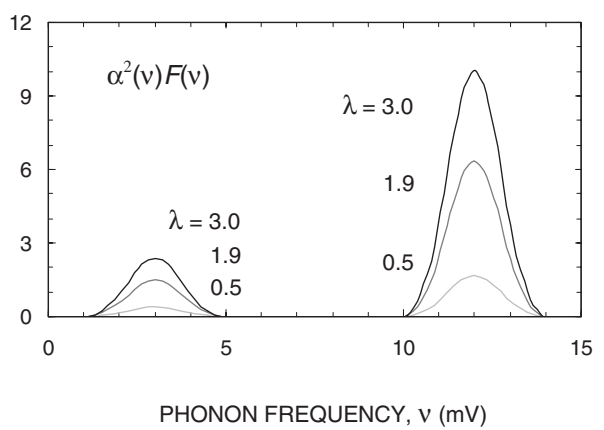

FIG. 1. Model spectra of the electron-phonon interaction. The peaks on the left and right are centered at $\nu_{0}=3$ and $12 \mathrm{mV}$, respectively.

bium $(\lambda=1.12)$ and indium $(\lambda=0.81)$, indicating that $\lambda$ $>0.8$ is likely to be the threshold in real materials. In Sec. VII, we show that weak-coupling superconductors with $\lambda$ $<0.8$ all have single-valued gap edges. These include indium, thallium (both listed in Ref. 23 as examples having double-valued gap edges), and aluminum. In Sec. VIII, we show that the shoulder in the temperature curve in mercury is an experimentally measurable feature that marks genuine departure from the BCS theory. We compare our results with gapless superconductivity in Sec. IX and give brief conclusions in Sec. X.

\section{MODEL SUPERCONDUCTOR}

It is instructive to begin with a model superconductor. The spectrum of electron-phonon interaction is usually chosen as either Einstein ${ }^{24,26}$ or Lorentzian;, 5 both have their limitations. In the former, $\Delta(\omega, T)$ bears little resemblance to the gap function in a real material. In the latter, the Lorentz curve has to be truncated to let $\alpha^{2}(\nu) F(\nu) \rightarrow 0$ when $\nu \rightarrow 0$, the price being that the curve no longer has a continuous derivative in $\nu$. So, in our model superconductor, $\alpha^{2}(\nu) F(\nu)$ is represented by a single peak of the square $B$ spline, ${ }^{27}$ which is continuous and has a continuous first order derivative, see Fig. 1. The center of the peak is located at $\nu=\nu_{0}$, $2 \leqslant \nu_{0} \leqslant 12 \mathrm{mV}$ and the base width of the peaks is always $4 \mathrm{mV}$. The height of the peak is adjusted to let $\lambda$, as defined in Eq. (2), vary between 0.5 and 3 to model different strengths of electron-phonon coupling. The Coulomb pseudopotential is fixed to let $\mu^{*}=0.1$, which falls in the range of Morel and Anderson. ${ }^{28}$

The procedure for solving the Eliashberg equations, in essence a specified method of iteration, has been detailed in the literature. ${ }^{9,29}$ In Fig. 2, we present the real part of the gap function solutions arising from the electron-phonon interactions in Fig. 1 at $T=0$. We drop from Fig. 2 the imaginary part of the functions for clarity. The electron integration cutoff frequency is chosen to be 50 and $100 \mathrm{mV}$ when $\nu_{0}=3$ and $12 \mathrm{mV}$, respectively, about ten and seven times the maximum phonon frequency, in accord with the accepted practice of solving the Eliashberg equations. ${ }^{30}$ We do not impose a predetermined value on the gap edge at $T=0$, which we will refer to as $\Delta_{0}$, but allow it to evolve naturally in the iteration 


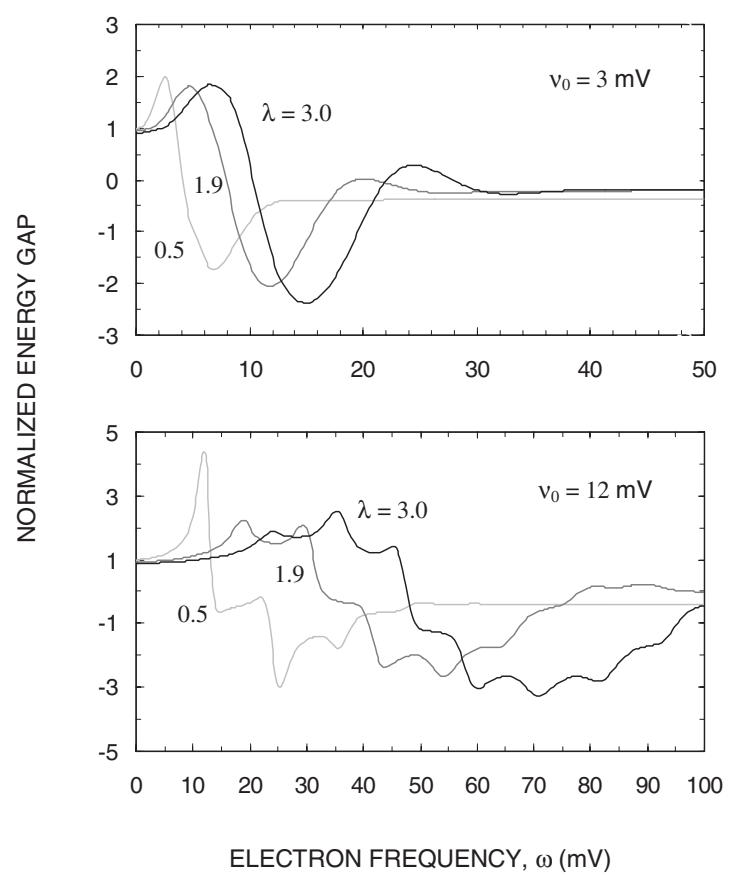

FIG. 2. Real part of normalized energy gap functions $(T=0)$ arising from the model electron-phonon interactions in Fig. 1; Coulomb pseudopotential $\mu^{*}=0.1$; the phonon peak is centered at $\nu_{0}$ $=3 \mathrm{mV}$ (upper) and $12 \mathrm{mV}$ (lower).

process as a result of the given values of $\lambda, \nu_{0}$, and $\mu^{*}$. We find that the gap function always varies slowly when $\omega \sim 0$, that is, $\Delta(\epsilon, 0) \simeq \Delta(0,0)$.

Next, we evaluate the gap function at a series of increasing temperatures. In order to expedite the process, we always start iteration with the converged gap function obtained at the previous temperature. Initially, the temperature is increased so that $T$ will reach $T_{c}$ after 30 steps on the conservative assumption that $2 \Delta_{0} / k_{B} T_{c}=3$. We reduce the temperature step to $1 / 4$ and $1 / 200$ of its initial size as the gap edge is reduced to $70 \%$ and $40 \%$ of its zero temperature value, respectively, in response to the steep descent of the gap edge when $T$ approaches $T_{c}$. At each temperature, we find the gap edge by solution of Eq. (1) and terminate calculations either when it becomes less than $0.005 \Delta_{0}$ or when Eq. (1) no longer has a nontrivial solution. To complete the curve, we follow a BCS-like form and assume that the real part of the gap edge is proportional to $\left(1-T / T_{c}\right)^{1 / 2}$ when $T \sim T_{c}{ }^{8}$, which enables us to find $T_{c}$ from the last value of the gap edge (or the last higher value when the gap edge is double valued) before calculation terminates. Numerical results of our calculations are presented in Table I.

We see from the upper part of Fig. 2 that, with $\nu_{0}$ $=3 \mathrm{mV}$, the gap functions always feature a significant initial peak followed by a significant dip. These peaks and dips tend to widen and move toward higher electron frequencies (energies) when $\lambda$ increases. We are reminded that in Fig. 2, the gap functions are normalized to the gap edge. We see from Table I that the gap edge also grows when $\lambda$ increases, so that, roughly speaking, $\lambda$ can be seen as a scaling factor for the gap function itself as well as for its argument. The gap-
TABLE I. Effect of the coupling constant on the gap edge, gapto-temperature ratio, and transition temperature using electronphonon interactions from the model in Fig. $1 ; \nu_{0}=3 \mathrm{mV}(12 \mathrm{mV}$ for value in parentheses and) and $\mu^{*}=0.1$.

\begin{tabular}{|c|c|c|c|c|}
\hline$\lambda$ & $\begin{array}{c}\Delta_{0} \\
(\mathrm{mV})\end{array}$ & $2 \Delta_{0} / k_{B} T_{c}$ & $\begin{array}{c}T_{c} \\
(\mathrm{~K})\end{array}$ & $\begin{array}{c}T_{c} \\
(\mathrm{~K})^{\mathrm{a}}\end{array}$ \\
\hline 0.5 & $0.76(3.99)$ & $3.62(3.59)$ & $0.5(2.6)$ & 0.4 (1.6) \\
\hline 1.9 & $12.6(63.4)$ & $4.97(5.27)$ & 5.9 (28) & 4.5 (19) \\
\hline 3.0 & $22.0(111)$ & $6.14(6.57)$ & $8.3(39)$ & $5.9(24)$ \\
\hline
\end{tabular}

${ }^{\mathrm{a}}$ From Eq. (3).

to-temperature ratio, $2 \Delta_{0} / k_{B} T_{c}$, grows from 3.62 to 6.14 when $\lambda$ increases from 0.5 to 3 , as is expected.

We see from the lower part of Fig. 2 that, with $\nu_{0}$ $=12 \mathrm{mV}$, the gap functions also feature a significant peak and dip, although some additional finer superimposed features emerge. The effects of $\lambda$ are also similar: it roughly scales the gap function and its argument and determines almost exclusively the value of the gap-to-temperature ratio. Indeed, $\nu_{0}$ has little effect on this ratio in Table I despite the fact that $T_{c}$ has increased approximately five, times compared with its value at $\nu_{0}=3 \mathrm{mV}$. This increase in $T_{c}$ was explored by Holcomb as a possible explanation for high temperature superconductivity in Ref. 9, where he added a peak of some electron-boson interaction with $\nu_{0}=1600 \mathrm{mV}$ to the spectrum of $\alpha^{2}(\nu) F(\nu)$ and found $T_{c}=118.1 \mathrm{~K}$.

The value of $T_{c}$ can also be estimated almost exclusively with $\lambda$. In order to highlight the significance of $\lambda$ in characterizing the electron-phonon system, important to our later discussions, as well as in showing that our model and numerical results are reasonable, we evaluate $T_{c}$ from the following Allen-Dynes formula: ${ }^{31}$

$$
T_{c}=\frac{\nu_{1}}{1.2} \exp \left[-\frac{1.04(1+\lambda)}{\lambda-\mu^{*}(1+0.62 \lambda)}\right],
$$

which is a variant of the McMillan formula ${ }^{32}$ and in which

$$
\nu_{1}=\frac{2}{\lambda} \int_{0}^{\omega_{M}} \alpha^{2}(\nu) F(\nu) d \nu
$$

measures the range of phonons involved in pairing electrons. We see from Table I that our model compares satisfactorily with the results in Eq. (3), a formula which is known to work well for real materials.

\section{GAP EDGE: DOUBLE VALUE AND SHOULDERLIKE STRUCTURE}

When solving the Eliashberg equations numerically, we divide the electron frequencies below the cutoff value into typically 200 divisions. In order to identify the doublevalued gap edges, we divide the electron frequencies in the first few divisions into a number of further subdivisions. Furthermore, we let $\omega=\Delta_{0} \times 10^{-4}$ be the minimum value of the electron frequencies instead of $\omega=0$, which is a singular point of the Eliashberg equations. The resulting temperature 


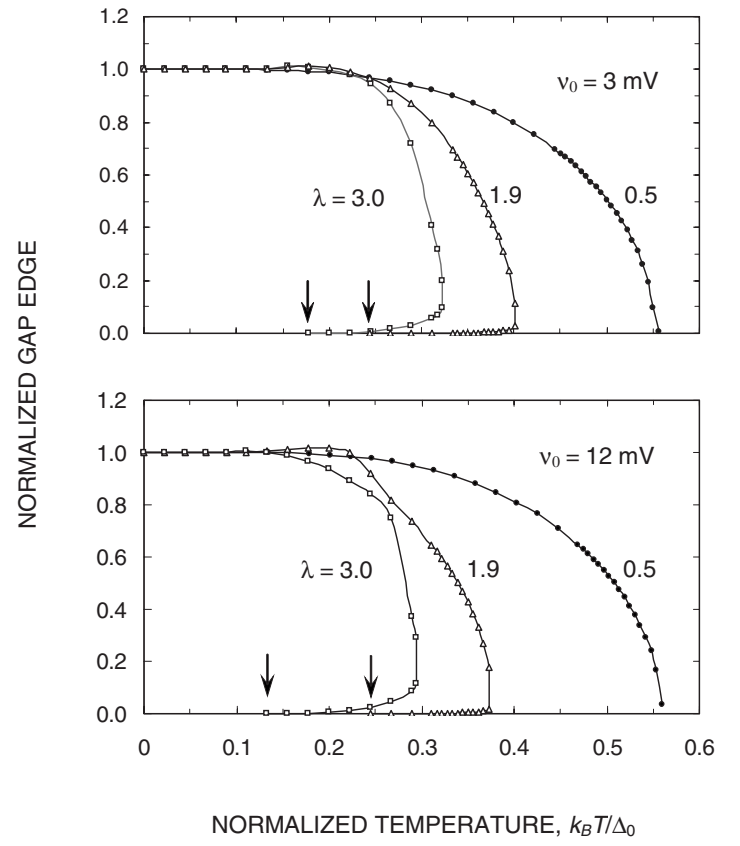

FIG. 3. Temperature curves of the gap edge of the model superconductor at various intensities of electron-phonon interaction. Both the gap edge and thermal energy are normalized by $\Delta_{0}$. Upper: with $\nu_{0}=3 \mathrm{mV}$, the gap edge becomes double valued when $k_{B} T$ $=0.244 \Delta_{0}$ at $\lambda=1.9\left(=0.178 \Delta_{0}\right.$ at $\lambda=3, T$ indicated by arrows $)$. Lower: with $\nu=12 \mathrm{mV}$, the gap edge becomes double valued when $k_{B} T=0.244 \Delta_{0}$ at $\lambda=1.9\left(=0.133 \Delta_{0}\right.$ at $\left.\lambda=3\right)$.

curves of the normalized gap edge for the $\nu_{0}=3 \mathrm{mV}$ model superconductor with $\lambda=0.5,1.9$, and 3 are presented in the upper part of Fig. 3. The temperature curve with $\lambda=0.5$ is always single valued and matches the BCS temperature curve well. The temperature curve with $\lambda=1.9$ has a gap edge which slightly exceeds its initial value $(=1.000)$ at $k_{B} T=0.067 \Delta_{0}$, reaches a peak $(=1.014)$ when $k_{B} T=0.178 \Delta_{0}$, and then drops faster than the $\lambda=0.5$ curve, with an overall shoulderlike appearance. It starts to become double valued at $k_{B} T=0.244 \Delta_{0}$. The temperature curve with $\lambda=3$ reaches its peak $(=1.015)$ at $k_{B} T=0.156 \Delta_{0}$ and then drops even more sharply. It starts to become double valued at $k_{B} T=0.178 \Delta_{0}$.

In the lower part of Fig. 3, we present the temperature curves for the $\nu_{0}=12 \mathrm{mV}$ model, also with $\lambda=0.5,1.9$, and 3 . The temperature curve with $\lambda=0.5$ is always single valued and matches the BCS temperature curve well. The temperature curve with $\lambda=1.9$ reaches its peak $(=1.015)$ when $k_{B} T$ $=0.178 \Delta_{0}$. It becomes double valued when $k_{B} T=0.244 \Delta_{0}$. The temperature curve with $\lambda=3$ has an insignificant peak $(=1.005)$ when $k_{B} T=0.111 \Delta_{0}$. It becomes double valued when $k_{B} T=0.133 \Delta_{0}$.

It is evident from Fig. 3 that the gap edge of the model superconductor may become double valued at a value of $\lambda$ somewhere between 0.5 and 1.9. To see this trend more clearly, we ran a numerical test, where we fix $\lambda$ at one of three values and let $\nu_{0}$ vary discretely between 2 and $12 \mathrm{mV}$ (interval $=0.5 \mathrm{mV}$, electron frequency cutoff $=100 \mathrm{mV}$ ). For each pair of $\lambda$ and $\nu_{0}$, we solve the Eliashberg equations over the whole range of temperatures when Eq. (1) has nontrivial solutions. In Fig. 4, we indicate the value of $\nu_{0}$ with an arrow

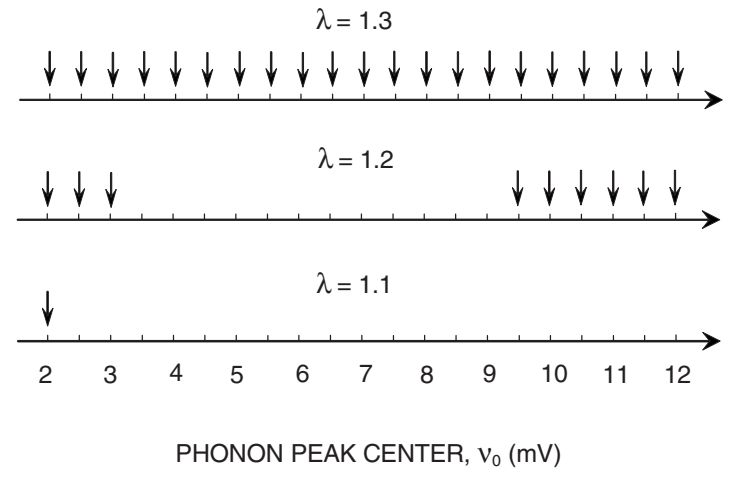

FIG. 4. Values of $\lambda$ and $\nu_{0}$, indicated by arrows, leading to double-valued gap edge of the model superconductor at some temperatures.

if at some temperatures the gap edge becomes double valued in the model superconductor with this $\nu_{0}$. It is apparent that while $\lambda$ has the dominant effect on the occurrence of the arrows, $\nu_{0}$ also has some effect, so that we may not be able to find a simple rule in terms of $\lambda$ to determine the distribution of the arrows. However, it appears that $\lambda \simeq 1$ may serve as a rough guide to warn us that in a real superconductor, the gap edge may acquire two distinct values at the same temperature. This value corresponds to moderately strong electron-phonon coupling.

Both the double-valued gap edge and shoulderlike structure in the temperature curve have a common origin in the Eliashberg-Nambu formalism. First, we consider the double values. As also observed by Holcomb, the weak-coupling $\Delta(\omega, T)$ may be approximated well by multiplying the zero temperature solution by the known dependence of the BCS gap, but this approximation clearly breaks down in the strong-coupling regime. Then, the gap function has a pronounced additional structure. ${ }^{9}$ For example, in Fig. 5, which shows it evaluated at $k_{B} T=0.222 \Delta_{0}$ (or $T=0.687 T_{c}$ ), there is a remarkably abrupt and significant drop of the gap function near $\omega=0$. It is similar to that reported at $T=0.7 T_{c}$ in Ref. 26, where Combescot calculated the gap function for a strongcoupling model superconductor both numerically and semianalytically in a radically different version of the Eliashberg formalism with imaginary electron frequencies. The diagrams in Fig. 5 clearly show that it is this very drop of the gap function near $\omega=0$ and specifically its near parabolic shape that dictate the double solutions of Eq. (1) and thus double values of the gap edge, as first highlighted by Leavens. ${ }^{23}$

Next, we address the shoulder in the gap edge versus temperature curves. In the lower part of Fig. 5, we show how, when $T$ increases, a significant drop of the gap function descends from above to intersect with the gray straight line twice to produce the double-valued gap edge: at smaller values of $T$, this drop will retreat upward, leaving a singlevalued solution to Eq. (1). However, while this retreating drop does not lead to double values of the gap edge, it implies that the gap edge also drops significantly, giving the shoulderlike structure in the temperature curve in the Eliashberg-Nambu formalism. Leavens used the expression $\omega^{2}$ to model the drop when it is low enough to be in touch 


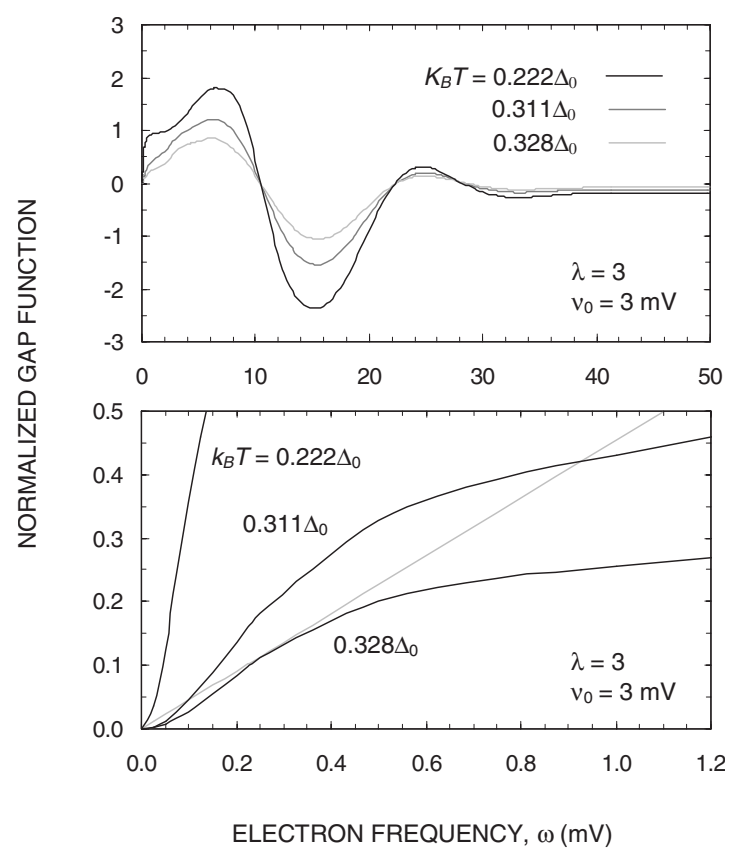

FIG. 5. Real part of gap functions of the model superconductor at temperatures approaching $T_{c}$. Each gap function is normalized by the corresponding value of $\Delta_{0}$. The effective phonon spectrum is a single peak in Fig. 1 with $\nu_{0}=3 \mathrm{mV}$ and $\lambda=3$. Upper: overall picture. Lower: close-up; values on gray line $=\omega$.

with the $\omega$ axis; see the text below Eq. (1). If we extend this idea by using an offset parabolic curve to model that retreating abrupt and significant drop of the gap function, then we find from the following pair of equations:

$$
\Delta=\omega^{2}+a, \quad \Delta=\omega
$$

a gap edge $\Delta=0.5+(0.25-a)^{1 / 2}$ where the offset $a$ becomes smaller the larger the value of $T$. Consequently, this gap edge grows with increasing $T$ and may even exceed $\Delta_{0}$ on account of the presence of large peaks of the gap functions at $\omega>0$ in Fig. 2, as long as the model in Eq. (4) is appropriate. This explains the peak (often $>\Delta_{0}$ ) of the temperature curves in Fig. 3.

From the Eliashberg equations, we may hope to find the most likely reason for the abrupt and significant drop of the gap function near $\omega=0$, which emerges at $T>0$ in strongcoupling superconductors but not in weak-coupling superconductors. There are four terms in each of the two kernels of the Eliashberg equations. ${ }^{9,29}$ Two of these four terms involve the Fermi-Dirac function $f\left(-\omega / k_{B} T\right)$, which takes the value 1 at $T=0$, when the gap function of any superconductor, strong and weak couplings alike, is virtually constant near $\omega=0$. The other two terms involve the function $f\left(\omega / k_{B} T\right)$, which vanishes at $T=0$. It is these two terms that appear to have brought in that abrupt and significant drop when $T$ is increased. Indeed, if we write the BCS gap equation into the Eliashberg-Nambu form with $\alpha^{2}(\nu) F(\nu)$, we have a kernel with just the two surviving terms at $T=0$, and the resultant temperature curve has neither double values nor

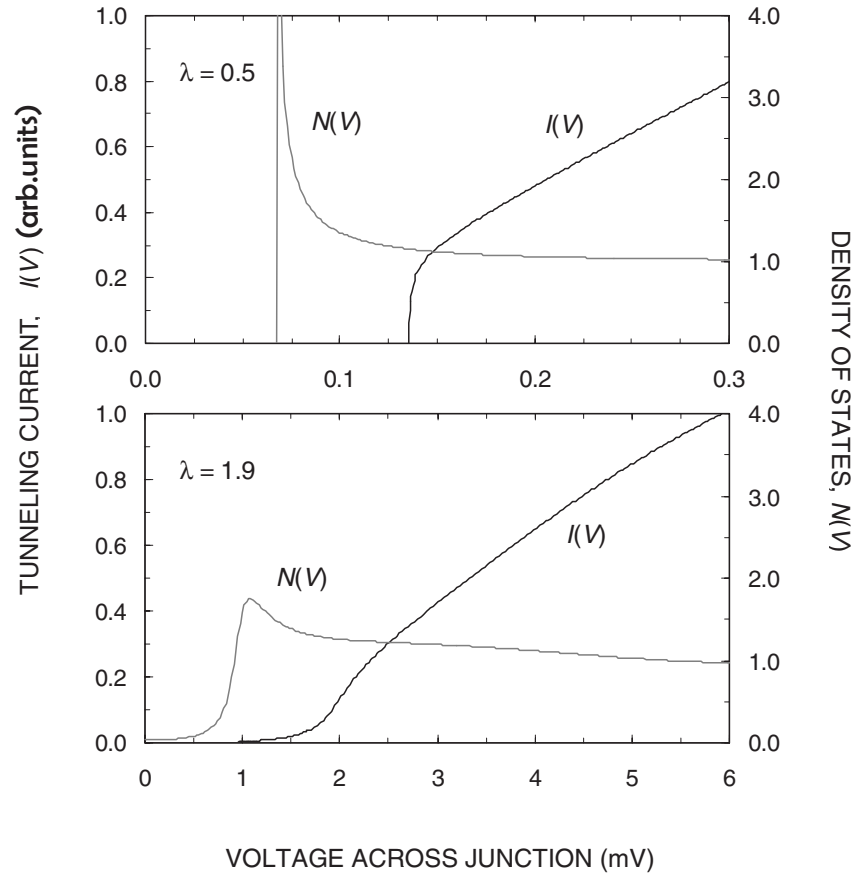

FIG. 6. Density of states and S-I-S tunneling current for the model superconductor; $k_{B} T=0.311 \Delta_{0} \quad\left(\right.$ or $T=0.54 T_{c}$ ) and $\nu_{0}$ $=3 \mathrm{mV}$.

the shoulderlike structure. Details will be reported in a forthcoming article.

\section{DENSITY OF STATES IN THE MODEL SUPERCONDUCTOR}

Knowledge of the gap function enables us to calculate the densities of states of the superconductive current carriers, defined as

$$
\frac{N(\omega)}{N(0)}=\mathfrak{R}\left[\frac{\omega}{\sqrt{\omega^{2}-\Delta^{2}}}\right],
$$

which we show in Fig. 6 for the model superconductor with $\lambda=0.5$ (upper plot) and 1.9 (lower plot) at a temperature $T$ $=0.54 T_{c}$; here, $\nu_{0}=3 \mathrm{mV}$. In the case of weak coupling, the density of states rises sharply when $\omega$ exceeds a certain value, which defines without much ambiguity the location of the energy gap edge. In the case of strong coupling, however, the density of states is finite right from zero frequency and rises slowly: the location of the gap edge is open to interpretation and purists would insist that the material is gapless. ${ }^{33-36}$ As Marsiglio and Carbotte ${ }^{24}$ remarked, the gap edge is often roughly associated with the frequency at which the peak in the density of states occurs. While this interpretation provides an unambiguous guide for us to locate the gap edge from the smooth curve with $\lambda=1.9$ at $T=0.98 T_{c}$ in Fig. 6, we do not have any sharp feature with which to identify the second gap edge.

Experimental values of the gap edge are found most directly from tunneling current-voltage characteristics, $I(V)$, which, according to McMillan and Rowell, are related to the 


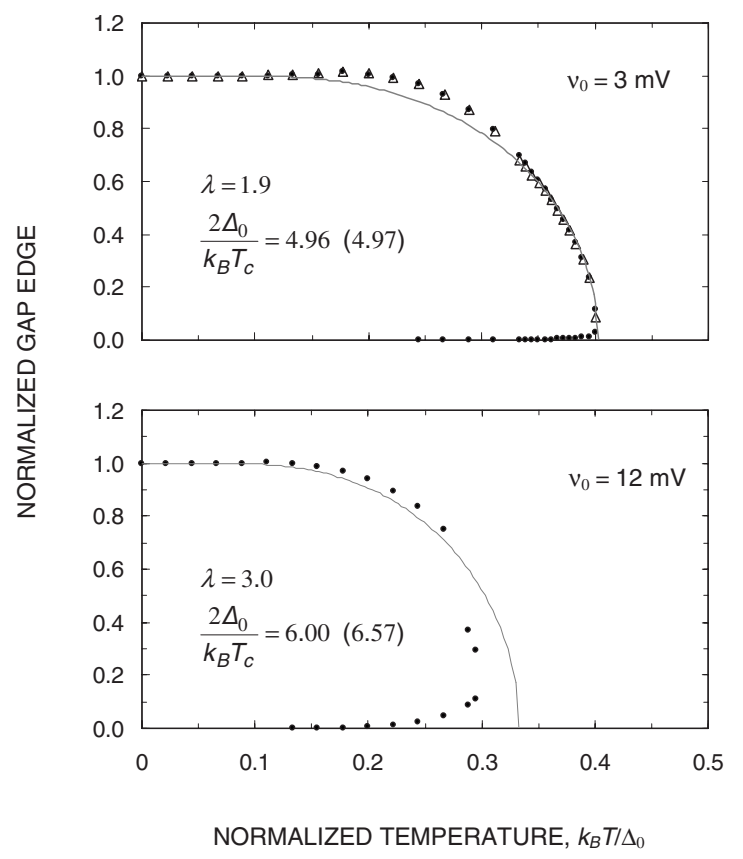

FIG. 7. Double-valued gap edge (dots), gap edge from tunneling current (triangles), and BCS temperature curve (gray line) in weakand strong-coupling model superconductors. The true order parameter vanishes at $T_{c}$ (for ratio in parentheses, $T_{c}$ is found from curve fitting with the dots).

electrode Eliashberg densities of states through the following formula:

$$
I(V)=\frac{1}{R} \int_{0}^{V} N(\omega) N(V-\omega) d \omega,
$$

for the case of tunneling between identical superconductor electrodes (S-I-S), where $I$ is the tunnel current, $V$ is the applied voltage difference between the electrodes, and $R$ is the junction resistance in the normal state. ${ }^{37}$ To calculate the tunnel current, we substitute the gap function from our numerical calculations into Eq. (5) and then substitute the resultant density of states into Eq. (6). As can be seen in Fig. 6, the current rises rapidly at applied voltage $V$ equal to twice the gap edge. This rise is sharp for the weak-coupling superconductors but more gradual for the strong-coupling superconductors. In practice, the value of the gap edge is found by a graphic method. ${ }^{37}$ In our case, we identify the gap edge when the slope of the tunneling current curve (conductance) reaches a maximum. We see from Fig. 7 that the gap so determined is always single valued; otherwise, it is almost identical to the gap edge found from Eq. (1). Apparently, the shoulderlike structure in the temperature curve is a genuine feature of a strong-coupling superconductor in the Eliashberg-Nambu formalism.

It is interesting that in Ref. 24, the gap edge of the model superconductor $(\lambda=100)$ becomes double valued when the temperature approaches $T_{c}$, yet it is enclosed completely by the BCS temperature curve, a condition similar to the two temperature curves with $\lambda=0.5$ and 3.0 in the lower part of Fig. 3. The reason is that, although the order parameter,
$\Delta(\omega, T)$, is by definition still nonzero up to $T_{c}$, nontrivial solutions of Eq. (1) cease to exist at $T=T^{*}<T_{c}^{23}$ : a sufficiently large area is therefore enclosed by the BCS curve to include the temperature curve of the double-valued gap edge arising from Eq. (1). In the lower part of Fig. 7, we show the double-valued gap edge of the $\lambda=3, \nu_{0}=12 \mathrm{mV}$ model superconductor, together with the BCS temperature curve; here, $T_{c}$ is where the order parameter, $\Delta(\omega, T)$, vanishes. The BCS temperature curve does not enclose the double-valued gap edge completely apparently because $\lambda$ is relatively small in our case. However, it is still clear that the double-valued gap edge and the non-BCS-like temperature curve go hand in hand in this situation.

It is worth mentioning that the lifetime broadening of the states in a strong-coupled superconducting alloy, $\mathrm{Pb}_{90} \mathrm{Bi}_{10}$, was previously calculated by Dynes et al. using the semiconductor model of a BCS superconductor to reproduce the current-voltage characteristic of a symmetric tunnel junction. ${ }^{38}$

\section{LEAD, MERCURY, AND BISMUTH-THALLIUM ALLOY}

In the literature, both experimental and theoretical data are surprisingly scarce for the temperature dependence curve of the superconductive energy gap edge in individual superconductive materials, particularly in the case of strong electron-phonon coupling. On the theoretical side, in 1963, the work of Nambu ${ }^{2}$ was developed and applied to lead $(\mathrm{Pb})$, a classical strong-coupling superconductor, by Schrieffer $e t$ al., with the phonon spectrum approximated by two Lorentzian peaks. ${ }^{5}$ In 1965 , lead was further investigated, also in the Eliashberg-Nambu formalism, by Scalapino and co-workers ${ }^{39,40}$ using an electron-phonon interaction calculated from the theoretical work by Harrison..$^{41}$ In 1969, lead was once more investigated by McMillan and Rowell who solved the Eliashberg equations with the electron-phonon interaction determined by inversion of tunneling data. ${ }^{37}$ In 1976, gap anisotropy in lead was studied by Tomlinson and Carbotte with the Eliashberg equations solved using electron-phonon interaction calculated from first principles. ${ }^{42}$ To our knowledge, the calculated temperature curve of the energy gap edge has not been published in the open literature from any of the above studies.

We have found seven discrete gap edge values for lead, calculated by Swihart et al. at $T>0$ and superimposed on experimental data by Gasparovic et al. in Ref. 43. These values are referred to but missing from Ref. 40. Later, the experimental data of Gasparovic et al., together with the seven calculated values, were copied back by Scalapino in Ref. 44 with no further details. The calculations apparently terminated when $T \sim 0.98 T_{c}$, with no evidence of a doublevalued gap edge. Indeed, the gap functions in Refs. 39 and 44 at $T=0.98 T_{c}$ vary slowly when $\omega \sim 0$, reminiscent of a weak-coupling superconductor: new calculations are necessary.

We sample the chart of $\alpha^{2}(\nu) F(\nu)$ in Ref. 31 and interpolate the sample values to other phonon frequencies by means of the cubic $B$ spline. ${ }^{27}$ We find $\lambda=1.42$ (between 1.33 and 1.55 in the literature ${ }^{37,42,45}$ ). We set the electron frequency 
TABLE II. Coupling constant, gap-to-temperature ratio, transition temperature, and starting temperature of double gap edges in real materials.

\begin{tabular}{ccccc}
\hline \hline & & \multicolumn{3}{c}{$T_{c}$} \\
& $\lambda$ & $2 \Delta_{0} / k_{B} T_{c}{ }^{\mathrm{a}}$ & $(\mathrm{K})^{\mathrm{a}}$ & $T / T_{c}{ }^{\mathrm{b}}$ \\
\hline $\mathrm{Hg}$ & 1.63 & $4.63(4.6)$ & $4.16(4.153)$ & 0.56 \\
$\mathrm{Bi}_{2} \mathrm{Tl}$ & 1.63 & $4.56(4.5)$ & $6.46(6.4)$ & 0.60 \\
$\mathrm{~Pb}$ & 1.42 & $4.42(4.38)$ & $7.24(7.193)$ & 0.78 \\
$\mathrm{Nb}$ & 1.12 & $3.79(3.8)$ & $9.22(9.50)$ & 0.97 \\
$\mathrm{In}$ & 0.81 & $3.73(3.6)$ & $3.22(3.4035)$ & 0.98 \\
$\mathrm{Tl}$ & 0.75 & $3.71(3.57)$ & $2.27(2.39)$ & \\
$\mathrm{Sn}$ & 0.74 & $3.70(3.5)$ & $3.60(3.722)$ & \\
$\mathrm{Al}$ & 0.43 & $3.53(3.3)$ & $1.12(1.140)$ & \\
\hline \hline
\end{tabular}

${ }^{\mathrm{a}}$ Experimental values (in parentheses) are from Ref. 46.

${ }^{\mathrm{b}}$ Gap edge becomes double valued when temperature $\geqslant T$.

cutoff at $60 \mathrm{mV}$ and find that at $T=0$, the calculated gap edge matches the experimental value of $1.37 \mathrm{mV}$ when $\mu^{*}=0.15$, compared with 0.12 in Ref. 37 and 0.13 in Ref. 45. At $T$ $>0$, we follow the procedure described in Sec. II and find $T_{c}=7.24 \mathrm{~K}\left(7.193 \mathrm{~K}\right.$ experimentally) and $2 \Delta_{0} / k_{B} T_{c}=4.42$ (4.38 experimentally), compared with $5.1 \mathrm{~K}$ by Eq. (3) and 4.52 in Ref. 30, see Table II. It is worth mentioning that in Ref. 46, the source of our experimental data, values of $\Delta_{0}$ and $T_{c}$ actually lead to a gap-to-temperature ratio of 4.40 rather than 4.38 .

In our calculations, the gap edge of lead starts to become double valued at $T=0.78 T_{c}$, when the gap function drops abruptly and significantly near $\omega=0$, similar to the abrupt and significant drop of the curve at $k_{B} T=0.222 \Delta_{0}$ in Fig. 5. The lower value of the gap edge remains small until close to $T_{c}$. On the other hand, the higher values of the gap edge are distinctively above the BCS curve. They are also slightly above the experimental data in Ref. 43. There is no peak in the temperature curve in the sense that it drops monotonically with a value always smaller than unity, see Fig. 8.

Mercury is another classical strong-coupling superconductor. In 1969, Hubin and Ginsberg measured the energy dependence of the tunneling density of states of superconducting mercury films at $0.4 \mathrm{~K} .{ }^{47}$ They extracted the phonon spectrum by McMillan inversion and solved the Eliashberg equations at $T=0$. There was no attempt to calculate the temperature dependence curve of the gap edge in spite of the fact that in an earlier exhaustive and careful experiment, the temperature curve of mercury was measured by the same group and published. ${ }^{25}$ Allen and Dynes listed $4.19 \mathrm{~K}$ as the transition temperature of mercury in the Eliashberg formalism, giving 4.57 (4.60 experimentally) as the gap-to-temperature ratio. ${ }^{31}$ However, no temperature curve was provided.

We have solved the Eliashberg equations also for mercury, with $\alpha^{2}(\nu) F(\nu)$ sampled from the chart in Ref. 47, finding $\lambda=1.63, \Delta_{0}=0.83 \mathrm{mV}$, and electron frequency cutoff at $45 \mathrm{mV}$, all in accord with the original specifications. Our calculated gap function at $T=0$ agrees well with the published curve in Ref. 47. We find $\mu^{*}=0.16$, compared with $\mu^{*}=0.11 \pm 0.04$ in Ref. 47. It is worth noting that, assuming
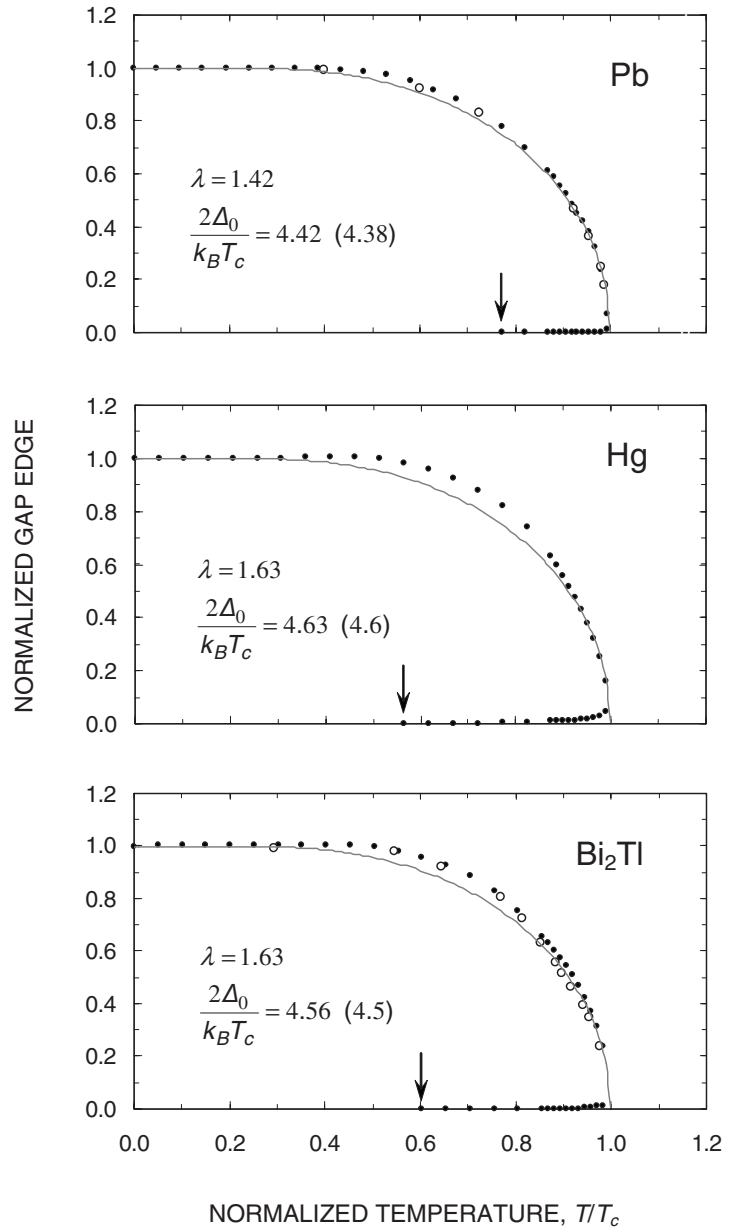

FIG. 8. Temperature dependence of the gap edge; the dots and solid lines are from numerical calculations and the BCS theory, respectively; experimental gap-to-temperature ratios are in parentheses. The gap edges start to become double valued when $T / T_{c}$ $\geqslant 0.78$ in lead [open circles from Swihart et al. (Ref. 40)], $\geqslant 0.56$ in mercury, and $\geqslant 0.60$ in the bismuth-thallium alloy [open circles from Vashishta and Carbotte (Ref. 48)], starting $T$ indicated by arrows.

$\alpha^{2}(\nu) F(\nu)$ to be proportional to $\nu^{4}$ or $\nu$ when $\nu \ll 1$, results in smaller (reduced by 0.07 ) and greater values of $\mu^{*}$, respectively, compared with the published case where $\alpha^{2}(\nu) F(\nu)$ is proportional to $\nu^{2}$ when $\nu \ll 1{ }^{47}$ We find $T_{c}=4.16 \mathrm{~K}$ (4.153 K experimentally) and $2 \Delta_{0} / k_{B} T_{c}=4.63$ (4.6 experimentally), compared with $3.7 \mathrm{~K}$ by Eq. (3) and 4.60 in Ref. 30.

In our calculations, the gap edge of mercury becomes double valued when $T \geqslant 0.56 T_{c}$, earlier than in lead. The lower values of the gap edge are also more significant compared with those in lead. Many of the higher values of the gap edge are significantly above the BCS curve, see Fig. 8. In particular, the temperature curve has a peak $(=1.007)$ at $T=0.463 T_{c}$, compared with the considerably lower value $(=0.971)$ of the BCS curve at $T=0.460 T_{c}$. In contrast, the experimental data of Bermon and Ginsberg match the BCS curve almost perfectly. ${ }^{25}$

The alloy $\mathrm{Bi}_{2} \mathrm{Tl}$ is one of the rare examples of strongcoupling superconductors with calculated temperature curves 
available in the literature. ${ }^{48}$ We sample $\alpha^{2}(\nu) F(\nu)$ from Ref. 48 , finding $\lambda=1.63,{ }^{31}$ and solve the Eliashberg equations with $\Delta_{0}=1.27 \mathrm{mV}$ and electron frequency cutoff at $75 \mathrm{mV}$. We find $\mu^{*}=0.19$ and $2 \Delta_{0} / k_{B} T_{c}=4.56$, compared with the experimental value of $4.5{ }^{48}$ We see from Fig. 8 that the gap edge becomes double valued when $T \geqslant 0.60 T_{c}$. The upper values of the gap edge compare well with the calculated results in Ref. 48, which are well above the BCS curve. In particular, our temperature curve has a peak $(=1.002)$ at $T$ $=0.453 T_{c}$, compared with the much lower BCS value $(=0.973)$ at $T=0.450 T_{c}$. There is no data point around $T$ $=0.450 T_{c}$ in Ref. 48. On the other hand, our nearest comparable value $(=0.980)$ at $T=0.554 T_{c}$ is close to the value in Ref. $48(=0.979)$ at $T=0.546 T_{c}$. We do not have experimental values of the gap edge for comparison.

\section{NIOBIUM AND INDIUM}

There was an interesting twist when the gap function in niobium was investigated. In 1976, Bostock et al. measured $\alpha^{2}(\nu) F(\nu)$ in tunneling experiments on $\mathrm{Nb}$ in the form of polycrystalline and high-purity, single crystal and solved the Eliashberg equations numerically. ${ }^{49}$ They found that typically, $\lambda=0.39$ and $\mu^{*}=-0.11$, that is, electrons should attract each other, and commented that either the electrons do behave like this or the Eliashberg formalism is inadequate. ${ }^{49}$ No temperature curve was provided. In 1977, Butler et al. found significantly different $\alpha^{2}(\nu) F(\nu)$ from theoretical calculations and neutron scattering, ${ }^{50}$ which leads to normal results with positive $\mu^{*} .{ }^{30}$ Arnold et al. concluded that the abnormal tunneling data in Ref. 49 arise from an anomalous thin weakly superconducting layer, probably $\mathrm{NbO}$, in the thermally oxidized $\mathrm{Nb}$ junctions. ${ }^{51}$

We solve the Eliashberg equations for niobium; the values of $\alpha^{2}(\nu) F(\nu)$ are sampled from the theoretical chart in Ref. 50. In our calculations, $\lambda=1.12\left(=1.20\right.$ in Ref. 50), $\Delta_{0}$ $=1.53 \mathrm{mV}$, and $\omega$ is cut off at $81 \mathrm{mV}$, all in accord with the listed data in Ref. 30 . We find $T_{c}=9.22 \mathrm{~K}(9.50 \mathrm{~K}$ experimentally), $\mu^{*}=0.39$, and $2 \Delta_{0} / k_{B} T_{c}=3.79$ (3.8 experimentally), compared with $T_{c}=1.2 \mathrm{~K}$ by Eq. (3), $\mu^{*}=0.27$, and $2 \Delta_{0} / k_{B} T_{c}=3.86$ in Ref. 30 . The gap edge becomes double valued when $T>0.97 T_{c}$ and the lower value of the doublevalued gap edge is always extremely small. The temperature curve declines monotonically with increasing $T$ and has no peak exceeding the gap edge value at $T=0$, see Fig. 9 .

We also sample the experimental values of $\alpha^{2}(\nu) F(\nu)$ for niobium in Ref. $50, \lambda=0.93$ and $\Delta_{0}=1.53 \mathrm{mV}$. We find $\mu^{*}$ $=0.15$ and $2 \Delta_{0} / k_{B} T_{c}=3.91$. The gap edge becomes double valued when $T>0.97 T_{c}$ with extremely small lower values. Furthermore, we solve the Eliashberg equations with $\alpha^{2}(\nu) F(\nu)$ from the controversial data in Ref. 49, $\lambda=0.39$ and $\Delta_{0}=1.53 \mathrm{mV}$. We find $\mu^{*}=-0.07$ and $2 \Delta_{0} / k_{B} T_{c}=3.65$. Surprisingly, we find that the gap edge becomes double valued when $T>0.99 T_{c}$, which is not in our experience with any other real material or model with such a low value of $\lambda$.

Indium was listed in Ref. 23 as an example having a double-valued gap edge. When solving the Eliashberg equations, we sample $\alpha^{2}(\nu) F(\nu)$ from the chart for indium in Ref.

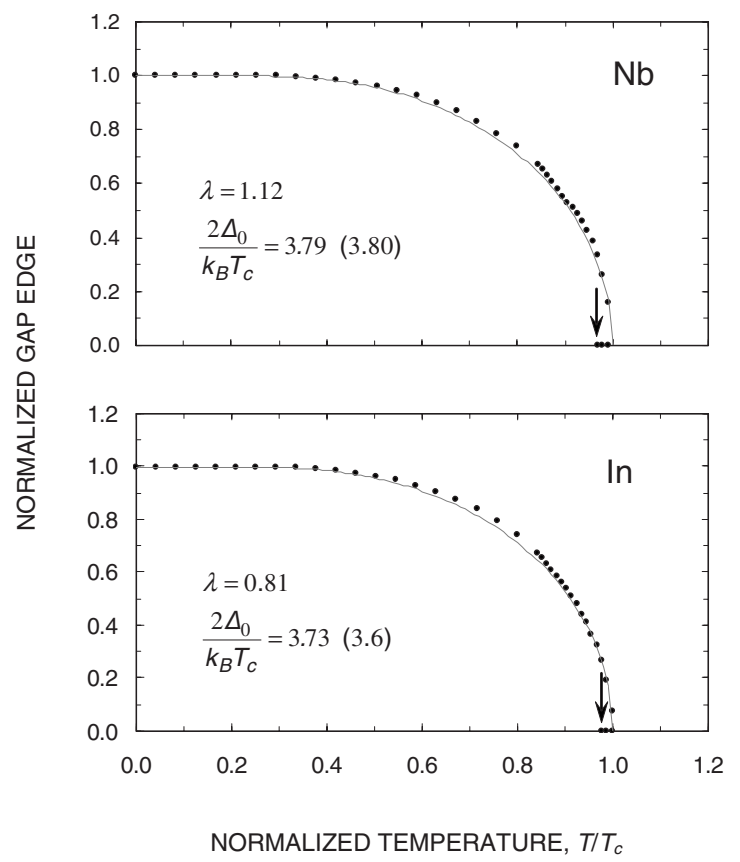

FIG. 9. Temperature dependence of the gap edge of niobium (theoretical phonons) (Ref. 50) and indium, $T / T_{c}=0.97$ and 0.98 , respectively, when the gap edge starts to become double valued; convention is identical to Fig. 8.

45 and find $\lambda=0.81$. In our calculations, $\omega$ is cut off at $80 \mathrm{mV}$ and $\mu^{*}=0.16$, compared with 0.125 in Ref. 45 . We find $T_{c}=3.22 \mathrm{~K}\left(3.4035 \mathrm{~K}\right.$ experimentally) and $2 \Delta_{0} / k_{B} T_{c}$ $=3.73$ ( 3.6 experimentally), compared with $2.3 \mathrm{~K}$ by Eq. (3) and 3.80 in Ref. 30 . The gap edge becomes double valued when $T=0.98 T_{c}$. The temperature curve also declines monotonically with increasing $T$, see Fig. 9.

\section{THALLIUM, TIN, AND ALUMINUM}

Thallium was listed in Ref. 23 as an example having a double-valued gap edge. We sample the Eliashberg function from the chart for $\mathrm{Tl}$ in Ref. 45 and find $\lambda=0.75$. In our calculations, $\omega$ is cut off at $60 \mathrm{mV}$ and $\mu^{*}=0.14$, compared with 0.127 in Ref. 45 . We find $T_{c}=2.30 \mathrm{~K}(2.39 \mathrm{~K}$ experimentally) and $2 \Delta_{0} / k_{B} T_{c}=3.71$ (3.57 experimentally), compared with $1.7 \mathrm{~K}$ by Eq. (3) and 3.76 in Ref. 30. The gap edge too is always single valued without any discernible shoulderlike structure.

Now, we solve the Eliashberg equations for tin, which is also listed in Ref. 23 as an example having a double-valued gap edge, $0.71<\lambda<0.80$ in Ref. 52. We sample $\alpha^{2}(\nu) F(\nu)$ from the chart in Ref. 52 and find $\lambda=0.74$. We cut $\omega$ off at $120 \mathrm{mV}$, in accord with the specifications in Ref. 52. Our gap function compares well in detail with the published result in Ref. 52. We find $\mu^{*}=0.15$, compared with 0.090 $\leqslant \mu^{*} \leqslant 0.145$ in Ref. 52. In our calculations, $T_{c}=3.60 \mathrm{~K}$ (3.722 K experimentally) and $2 \Delta_{0} / k_{B} T_{c}=3.70$ (3.5 experimentally), compared with $2.5 \mathrm{~K}$ by Eq. (3) and 3.71 in Ref. 30 . The gap edge is always single valued and without any shoulderlike structure, see Fig. 10. 


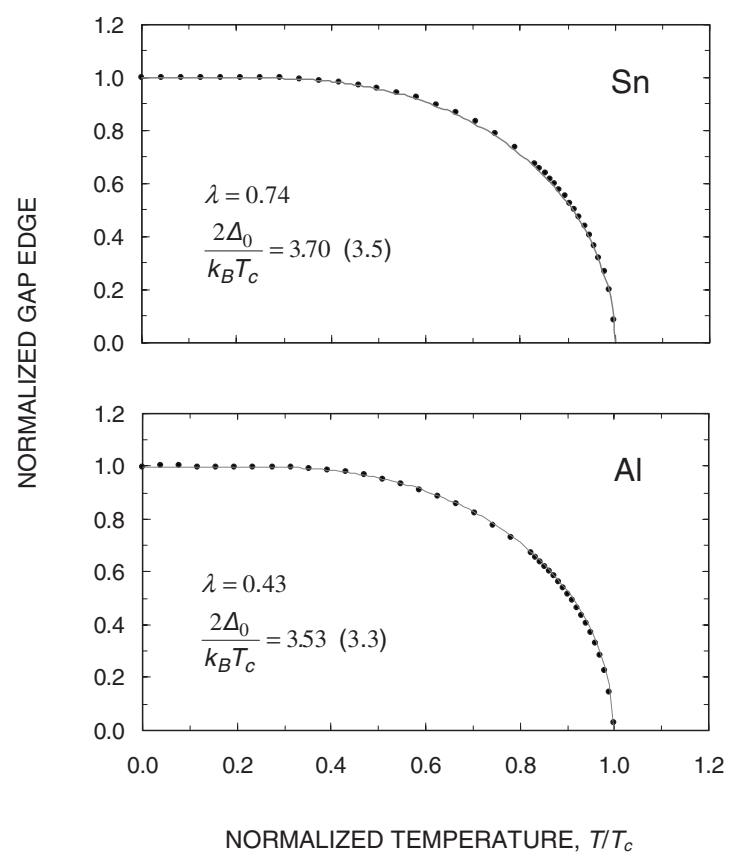

FIG. 10. Temperature dependence of the gap edge of tin and aluminum; convention is identical to Fig. 9.

We sample the values of $\alpha^{2}(\nu) F(\nu)$ for aluminum $(\lambda$ $=0.43$ ) from the chart in Ref. 53, which is apparently from theoretical calculations by Leung et al. ${ }^{54}$ We cut $\omega$ off at $150 \mathrm{mV}$ and find $\mu^{*}=0.1757, T_{c}=1.116 \mathrm{~K}(1.140 \mathrm{~K}$ experimentally), and $2 \Delta_{0} / k_{B} T_{c}=3.53$ (3.3 experimentally), compared with $\mu^{*}=0.1472, T_{c}=1.18 \mathrm{~K}$, and $2 \Delta_{0} / k_{B} T_{c}=3.54$ in Ref. 30. The gap edge again is always single valued without any shoulderlike structure.

\section{DENSITIES OF STATES AND TUNNELING CHARACTERISTICS IN TIN AND MERCURY}

We study the densities of states, as well as the tunneling characteristics, in two contrasting materials, tin and mercury, by following the procedure described in Sec. IV. We show the results in Fig. 11, which can be compared with Fig. 6. It is apparent that while in tin the gap edge can be determined from the sharp rise in tunneling current without much ambiguity, in mercury, the situation is compromised by a significant current flow at voltages below the main current rise, which itself is not sharp. This highlights a genuine and significant difference between weak- and strong-coupling superconductors in the Eliashberg-Nambu formalism.

In Fig. 12, we compare results of two interpretations of the tunneling current in mercury. The lower value of the gap edge, arising from Eq. (1), vanishes when we let the value of the energy gap edge be the voltage where the slope of the current (conductance) reaches its maximum. Otherwise, there is little difference between the value derived from Eq. (1) and that based on the tunneling current. In particular, there is no substantial difference in the value of $T_{c}$. In a real material, Eq. (1) is not at an obvious disadvantage despite offering no nontrivial solution when the order parameter still exists. ${ }^{24}$

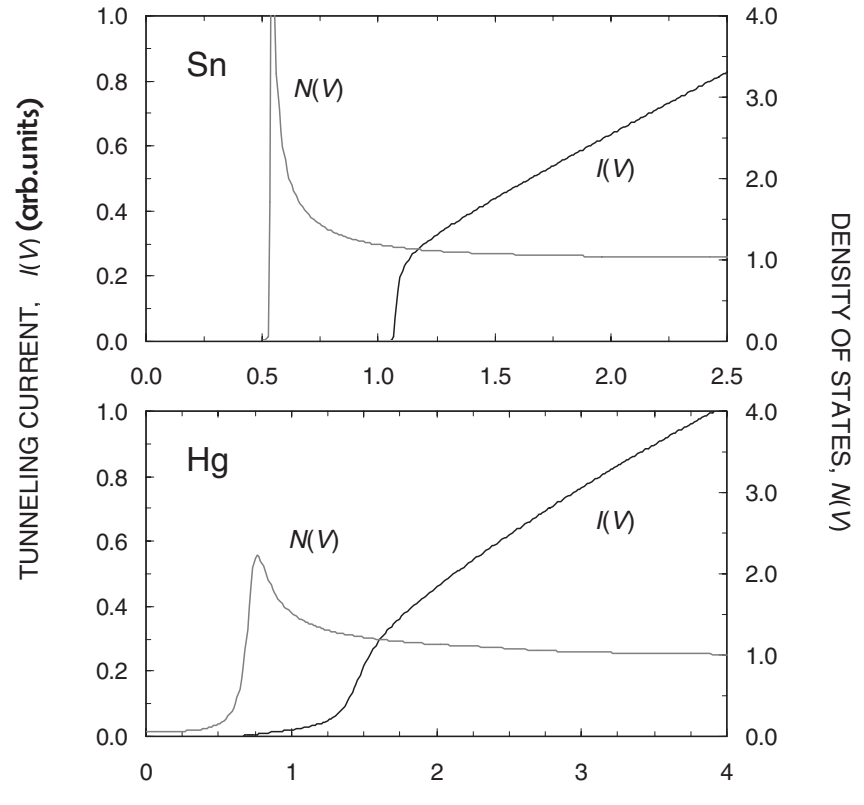

VOLTAGE ACROSS JUNCTION (mV)

FIG. 11. Density of states and S-I-S tunneling current for tin and mercury; $k_{B} T=0.311 \Delta_{0}$ in both cases.

The substance of Fig. 12 appears to be the shoulderlike structure of the temperature curve from the EliashbergNambu formalism: that is, values of the gap edge in this curve lie significantly above the BCS curve regardless of whether Eq. (1) or the tunneling current interpretation is employed. On the other hand, we know from experiment that in mercury the variation of the gap edge against temperature is predicted almost precisely by the BCS theory. ${ }^{25}$

We are reminded that the shoulderlike structure in the temperature curve in the Eliashberg-Nambu formalism may not be simply dismissed as an artifact. It is apparent from the experimental data ${ }^{43}$ that in lead, values of the gap edge do lie above the BCS temperature curve and compare fairly well with the temperature curve from the Eliashberg-Nambu formalism. Further experimental verification appears to be necessary in, for example, $\mathrm{Bi}_{2} \mathrm{Tl}$, where the BCS theory and

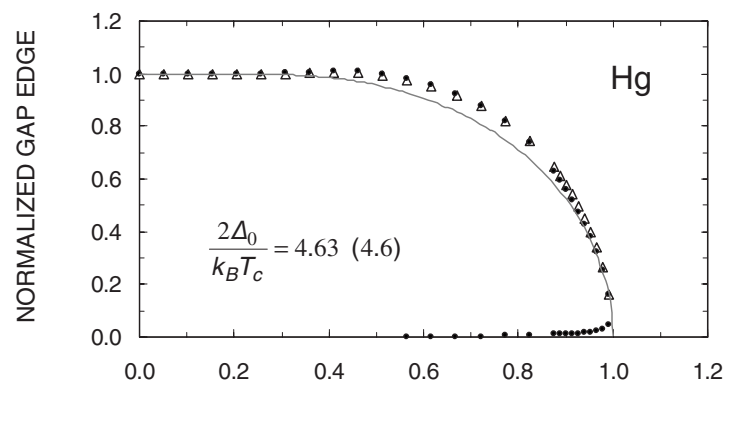

NORMALIZED TEMPERATURE, $T / T_{c}$

FIG. 12. Double-valued gap edge (dots), gap edge from tunneling current (triangles), and BCS temperature curve (gray line) of mercury. Here, we use the tunneling current to find $T_{c}$, and hence the gap-to-temperature-ratio (experimental value in parentheses). 
Eliashberg-Nambu formalism again differ significantly with respect to their predictions of the shape of the temperature curve. $^{48}$

\section{DISCUSSION}

Abrikosov and Gorkov predicted that magnetic impurities would cause a drastic alteration in the density of states of superconductors, leading to gapless superconductivity. ${ }^{33,34}$ This prediction was verified experimentally by Reif and Woolf, ${ }^{35}$ Edelstein ${ }^{36}$ and a number of other authors, mainly by means of tunneling, with mixed results with respect to consistency between theory and experiment.

In many ways, such gapless superconductivity can be compared with the strongly coupled Eliashberg-Nambu state. For example, the former arises from a theoretical argument that the quasiparticle states decay in the presence of magnetic impurities, ${ }^{33}$ whereas the latter is also attributed to breakdown of the quasiparticle approximation. ${ }^{23}$ The resultant densities of states are remarkably similar in both cases, see diagrams in Refs. 34 and 36 and Figs. 6 and 11 here. This appears to have been noticed by Dynes et al. when they in effect followed the practice in gapless superconductivity ${ }^{34}$ of introducing a complex frequency, $\omega+i \Gamma$, into the BCS theory to simulate the temperature broadening of the density of states in strong-coupling superconductors. ${ }^{38}$

We can also interpret as gapless superconductivity the situation where Eq. (1) does not have a nontrivial solution while the true order parameter still exists. Indeed, as long as $\Delta(\omega, T)$ is finite, we are invited to define the following three regimes:

$$
\mathfrak{R}[\Delta(\omega, T)] \begin{cases}>\omega, & \text { gapped } \\ =\omega, & \text { gap edge } \\ <\omega, & \text { gapless. }\end{cases}
$$

These three regimes exist as long as Eq. (1) has two nontrivial solutions at a given temperature. The gapless state is not associated with a sharp feature in the tunneling current, and hence is more difficult to demonstrate than the other two conditions. However, in the model superconductor in the lower part of Fig. 7, the gapless state alone exists when $k_{B} T>0.3 \Delta_{0}$ and is apparently subject to direct detection.

In real strong-coupling superconductive materials, the gapless state does not exist alone in any significant range of temperatures. However, while a gapless state in such materials might be elusive, we may detect the shoulderlike structure in the temperature curve, which has the same origin as the double-valued gap edge. Indeed, we see from Fig. 8 that in $\mathrm{Pb}, \mathrm{Hg}$, and $\mathrm{Bi}_{2} \mathrm{Tl}$, the shoulders are distinctively above the BCS curve and are certainly detectable experimentally. So far, the experimental results have been mixed with respect to their consistency with the Eliashberg-Nambu formalism: they are seemingly consistent in $\mathrm{Pb}$ (Ref. 43) but definitely inconsistent in $\mathrm{Hg} .{ }^{25}$ It remains to be seen whether in $\mathrm{Bi}_{2} \mathrm{Tl}$ the temperature curve of the gap edge has a shoulder in accordance with the Eliashberg-Nambu formalism.

Leavens pointed out that the quasiparticle approximation is a basic assumption of the original BCS theory. ${ }^{23}$ We wish to point out that the Eliashberg-Nambu formalism is also founded on Landau's idea of a Fermi liquid, ${ }^{55}$ together with Migdal's adiabatic theorem. ${ }^{4}$ The former justifies how a fundamental treatment developed originally for a Fermi gas, for example, by labeling a microscopic entity (namely, an electron) with its momentum and spin, can be applied to a Fermi liquid where the interaction between microscopic entities (that is, elementary excitations or quasiparticles) can no longer be ignored. The latter can be used to justify the application of Landau's idea to the pairing interaction that leads to superconductivity. ${ }^{44}$ The quasiparticle approximation breaks down with large $\lambda$ and at relatively high $T$, coincident with the appearance of the double-valued gap edge and the shoulder in and only in the Eliashberg-Nambu formalism. The question is why this formalism is so different from the BCS theory.

In the Eliashberg-Nambu formalism, the one-electron self-energy is calculated by what amounts to second-order self-consistent perturbation theory. ${ }^{44}$ In contrast, the BCS theory arises from the variational approach, ${ }^{8}$ which is well known to be tolerant of errors in the trial function. In the case of $\mathrm{Hg}$, the BCS theory proves itself to be better than the Eliashberg-Nambu theory. If the experimental temperature curve in $\mathrm{Bi}_{2} \mathrm{Tl}$ turns out again to be in favor of the $\mathrm{BCS}$ theory, then we might have to consider carefully the limitations of the Eliashberg-Nambu formalism when applied to strong-coupling superconductors.

\section{SUMMARY AND CONCLUSIONS}

We have elaborated the discovery of Leavens that in the Eliashberg-Nambu formalism, the edge of the superconductive energy gap can have two distinct values at the same temperature. We have solved the Eliashberg equations numerically for a model material, a range of elemental superconductors, including $\mathrm{Al}, \mathrm{Sn}, \mathrm{Tl}, \mathrm{Nb}, \mathrm{In}, \mathrm{Pb}$, and $\mathrm{Hg}$, and one alloy, $\mathrm{Bi}_{2} \mathrm{Tl}$. We have confirmed the discovery of Leavens when $\lambda>0.8$; otherwise, the gap edge will be single valued.

We have also elaborated the discovery by Marsiglio and Carbotte that when $\lambda=100$, the temperature dependence of the gap edge in the Eliashberg-Nambu formalism deviates significantly from the prediction by the BCS theory. We have found that in real strong-coupling superconductors, for example, $\mathrm{Pb}, \mathrm{Hg}$, and $\mathrm{Bi}_{2} \mathrm{Tl}$, with moderately large values of $\lambda$, there is a shoulderlike structure in the temperature dependence curve of the gap edge, marking significant deviation from the BCS temperature curve. We have found that values of the gap edge at the peak of the shoulder may even exceed its value at $T=0$.

We have proved that the double values of the gap edge and the shoulderlike structure have the same origin: both arise from a sudden and significant drop of the gap function near $\omega=0$. We have found vastly different densities of states between weak- and strong-coupling superconductors. We also find numerically from these densities of states the tunneling current, according to the simple McMillan prescription, in an effort to reproduce the experimental measurement of the gap edge. We see from this measurement that the 
shoulderlike structure is still evident. Both determinations are genuine signs indicating significant departure of the Eliashberg-Nambu formalism from the BCS theory.

We have compared the density of states in strongcoupling superconductors with the density of states in gap- less superconductors in the presence of magnetic impurities. We conclude that further experiment is needed, particularly in $\mathrm{Bi}_{2} \mathrm{Tl}$, in order to expose possible limitations of the Eliashberg-Nambu formalism in real strong-coupling superconductive materials. *xhz@qub.ac.uk

${ }^{1}$ G. M. Eliashberg, Sov. Phys. JETP 11, 696 (1960).

${ }^{2}$ Y. Nambu, Phys. Rev. 117, 648 (1960).

${ }^{3}$ L. P. Gokov, Sov. Phys. JETP 7, 505 (1958).

${ }^{4}$ A. B. Migdal, Sov. Phys. JETP 7, 996 (1958).

${ }^{5}$ J. R. Schrieffer, D. J. Scalapino, and J. W. Wilkins, Phys. Rev. Lett. 10, 336 (1963); D. J. Scalapino, J. R. Schrieffer, and J. W. Wilkins, Phys. Rev. 148, 263 (1966).

${ }^{6}$ Y. Wada, Phys. Rev. 135, A1481 (1964).

${ }^{7}$ V. Ambegaokar and L. Tewordt Phys. Rev. 134, A805 (1964).

${ }^{8}$ J. Bardeen, L. N. Cooper, and J. R. Schrieffer, Phys. Rev. 108, 1175 (1957).

${ }^{9}$ M. J. Holcomb, Phys. Rev. B 54, 6648 (1996).

${ }^{10}$ Han-Yong Choi, Phys. Rev. Lett. 81, 441 (1998).

${ }^{11}$ G. A. Ummarino and R. S. Gonnelli, Phys. Rev. B 66, 104514 (2002).

${ }^{12}$ P. Monthoux and G. G. Lonzarich, Phys. Rev. B 59, 14598 (1999).

${ }^{13}$ A. Knigavko and F. Marsiglio, Phys. Rev. B 64, 172513 (2001).

${ }^{14}$ H. J. Choi, D. Roundy, H. Sun, M. L. Cohen, and S. G. Louie, Nature (London) 418, 758 (2002).

${ }^{15}$ O. V. Dolgov, R. S. Gonnelli, G. A. Ummarino, A. A. Golubov, S. V. Shulga, and J. Kortus, Phys. Rev. B 68, 132503 (2003).

${ }^{16}$ O. V. Dolgov, R. K. Kremer, J. Kortus, A. A. Golubov, and S. V. Shulga, Phys. Rev. B 72, 024504 (2005).

${ }^{17}$ Tae-Ho Park and Han-Yong Choi, Phys. Rev. B 65, 052504 (2002).

${ }^{18}$ K. Kuroki, Y. Tanaka, and R. Arita, Phys. Rev. Lett. 93, 077001 (2004).

${ }^{19}$ S. K. Bose, T. Kato, and O. Jepsen, Phys. Rev. B 72, 184509 (2005).

${ }^{20}$ O. V. Dolgov, I. I. Mazin, A. A. Golubov, S. Y. Savrasov, and E. G. Maksimov, Phys. Rev. Lett. 95, 257003 (2005)

${ }^{21}$ F. Zipoli, M. Bernasconi, and G. Benedek, Phys. Rev. B 74, 205408 (2006).

${ }^{22}$ E. Schachinger, D. Neuber, and J. P. Carbotte, Phys. Rev. B 73, 184507 (2006).

${ }^{23}$ C. R. Leavens, Phys. Rev. B 29, 5178 (1984).

${ }^{24}$ F. Marsiglio and J. P. Carbotte, Phys. Rev. B 43, 5355 (1991).

${ }^{25}$ S. Bermon and D. M. Ginsberg, Phys. Rev. 135, A306 (1964).

${ }^{26}$ R. Combescot, Phys. Rev. B 51, 11625 (1995).

${ }^{27} \mathrm{H}$. Prautzsch, W. Boehm, and M. Paluszny, Bźier and B-Spline Techniques (Springer, Berlin, 2000), p. 86.

${ }^{28}$ P. Morel and P. W. Anderson, Phys. Rev. 125, 1263 (1962).
${ }^{29}$ X. H. Zheng and D. G. Walmsley, Phys. Rev. B 76, 224520 (2007).

${ }^{30}$ B. Mitrović, H. G. Zarate, and J. P. Carbotte, Phys. Rev. B 29, 184 (1984).

${ }^{31}$ P. B. Allen and R. C. Dynes, Phys. Rev. B 12, 905 (1975).

${ }^{32}$ W. L. McMillan, Phys. Rev. 167, 331 (1968).

${ }^{33}$ A. A. Abrikosov and L. P. Gorkov, Sov. Phys. JETP 12, 1243 (1961).

${ }^{34}$ J. C. Phillips, Phys. Rev. Lett. 10, 96 (1963)

${ }^{35}$ F. Reif and M. A. Woolf, Phys. Rev. Lett. 9, 315 (1962).

${ }^{36}$ A. S. Edelstein, Phys. Rev. Lett. 19, 1184 (1967).

${ }^{37}$ W. L. McMillan and J. M. Rowell, in Superconductivity, edited by R. D. Parks (Dekker, New York, 1969), Vol. 1, p. 561.

${ }^{38}$ R. C. Dynes, V. Narayanamurti, and J. P. Garno, Phys. Rev. Lett. 41, 1509 (1978)

${ }^{39}$ D. J. Scalapino, Y. Wada, and J. C. Swihart, Phys. Rev. Lett. 14, 102 (1965)

${ }^{40}$ J. C. Swihart, D. J. Scalapino, and Y. Wada, Phys. Rev. Lett. 14, 106 (1965).

${ }^{41}$ W. A. Harrison, Phys. Rev. 131, 2433 (1963).

${ }^{42}$ P. G. Tomlinson and J. P. Carbotte, Phys. Rev. B 13, 4738 (1976).

${ }^{43}$ R. F. Gasparovic, B. N. Taylor, and R. E. Eck, Solid State Commun. 4, 59 (1965).

${ }^{44}$ D. J. Scalapino, in Superconductivity, edited by R. D. Parks (Dekker, New York, 1969), Vol. 1, p. 449.

${ }^{45}$ R. C. Dynes, Phys. Rev. B 2, 644 (1970).

${ }^{46}$ C. Kittel, Introduction to Solid State Physics, 6th ed. (Wiley, New York, 1986), Chap. 12.

${ }^{47}$ W. N. Hubin and D. M. Ginsberg, Phys. Rev. 188, 716 (1969).

${ }^{48}$ P. Vashishta and J. P. Carbotte, Phys. Rev. B 10, 2789 (1974).

${ }^{49}$ J. Bostock, V. Diadiuk, W. N. Cheung, K. H. Lo, R. M. Rose, and M. L. A. MacVicar, Phys. Rev. Lett. 36, 603 (1976).

${ }^{50}$ W. H. Butler, H. G. Smith, and N. Wakabayashi, Phys. Rev. Lett. 39, 1004 (1977).

${ }^{51}$ G. B. Arnold, J. Zasadzinski, and E. L. Wolf, Phys. Lett. 69A, 136 (1978).

${ }^{52}$ J. M. Rowell, W. L. McMillan, and W. L. Feldmann, Phys. Rev. B 3, 4065 (1971).

${ }^{53}$ J. Zasadzinski, D. M. Burnell, E. L. Wolf, and G. B. Arnold, Phys. Rev. B 25, 1622 (1982).

${ }^{54}$ H. K. Leung, J. P. Carbotte, D. W. Taylor, and C. R. Leavens, Can. J. Phys. 54, 1585 (1976).

${ }^{55}$ L. D. Landau, Sov. Phys. JETP 3, 920 (1957). 